\title{
The Integration of Communication Technology and Wireless Communication in the Internet of Things
}

\author{
Zihao Fu \\ School of North China Electric Power University, Beijing 102206, China \\ 18810555907@163.com
}

\begin{abstract}
Keywords: Internet of things; Communications technology; Mobile network; Cloud computing
\end{abstract}
\begin{abstract}
Internet of things technology as a new technology, pursue all devices connected to the Internet. In order to achieve the desired object recognition, positioning, monitoring and other needs. At present, the technology of Internet of things is mainly short distance communication, including NFC, Bluetooth and other common technology. Long distance communications still have to rely on 3G, 4G Technology, but there are still some problems. As an emerging technology, The Internet of things plays an important role in the future of Intelligent Earth. It is necessary to analyze and discuss it, especially for the mobile communication technology of the Internet of things.
\end{abstract}

\section{Introduction}

Internet of things technology, refers to a variety of information sensing devices, such as infrared sensors, radio frequency identification device (RFID), global positioning system (GPS), laser scanning and other devices and the Internet together to form a huge network. Its purpose is to allow all devices can be connected with the Internet, and real-time automatic identification of the required objects, positioning, tracking, monitoring sand real-time feedback. After the computer technology, Internet and mobile communication network, the Internet of things has become the world's third wave of information industry. The concept of "Internet of things" is a kind of new thinking. The past has always been the separation of infrastructure such as buildings, roads and data transmission devices such as computers and communications equipment. After the concepts of the Internet of things come out, the two will be integrated into a unified infrastructure.

In 1999, the concept of "Internet of things" was put forward, and it was proposed that all the items can be connected through the information transmission equipment such as radio frequency identification, so as to realize intelligent identification and management.In fact, the "Internet of things" technology is the extension and extension of Internet technology, is the extension and expansion of information exchange and communications to any object and object. Therefore, the development of the Internet of things is an inevitable choice for mobile network operators, which has a very broad market prospects. The application of Internet of things has brought forward many new demands for the existing communication system. Therefore, how to make full use of the existing communication network operation and maintenance, enhance the competitiveness of the communication network in the future, has become an important issue in front of mobile network operators. Due to the traditional wireless communication network cannot meet the needs of the Internet of things for data transmission, many aspects of early Internet applications such as deployment and application areas have been limited, it is difficult to cooperate between different terminals and terminals. With the development of wireless communication technology, cloud computing, cloud storage and other technologies, end-to-end wireless network will become inevitable. Wireless communication network will become the foundation of the Internet of things system, the mobile communication terminal can also be combined with the Internet of things terminal. 


\section{The Development of Internet of Things}

Internet of Things Short Distance Communication Technology and Cloud Computing. The communication technology of the Internet of things mainly includes two aspects, namely the sensor network communication technology and the telecommunication transmission network communication technology. The sensor network is also known as the end of the network, the use of communication technology is mainly for short-range communications technology, including RFID technology, NFC, ZigBee, Bluetooth, etc. The telecommunication transmission network is also called the core bearer network, which includes the communication technology between the sensor network and the transmission network and the communication technology of the telecommunication transmission network. Telecommunication network technology includes all optical network, SDH and other wired communication technology, 2G, 3G, LTE and other mobile communications technology is developing. Because of the ubiquitous characteristics of Internet of things technology, mobile wireless communication technology is more important.

The existing networking communication technology includes RFID communication technology, NFC technology and Bluetooth technology and so on.

Among them, RFID (Radio Frequency Identification) technology is a short distance wireless communication technology based on radio frequency, also known as radio frequency identification technology or electronic tag. RFID is a simple wireless transmission system, which is composed of three parts: tag, reader and antenna. RFID technology has many advantages, such as easy to read, large data capacity, fast identification speed, long service life, good security, wide application, etc. NFC (Near Field Communication) is a kind of high frequency short distance wireless communication technology, which can be used to communicate and exchange data between two kinds of devices in the $10 \mathrm{~cm}$. NFC technology for the integration of non-contact RF technology and interconnect technology, RF signal through target recognition and access to relevant data, can automatically set up a wireless network, mobile equipment, electronic products and other equipment in short distance communication. NFC can make two devices do not need to connect to complete the exchange of information, just close to each other. It has three advantages: one is the use of private communication. RF range is small so it has high security. Signal attenuation technology, with high speed, high bandwidth and low consumption characteristics. Besides, it can be two-way connection and identification.

Bluetooth technology is a kind of support equipment in short distance $(10 \mathrm{~m})$ wireless communication technology, which has no linear, openness, compatibility, mobility, anti-jamming, power consumption and cost are low, the interconnection of each equipment in the network close to achieve seamless sharing of resources.

ZigBee technology is a short distance, low power consumption rate and cost of two-way wireless communication technology, used for the transmission of data between electronic devices and the typical periodic data transmission applications. It has the advantages of low transmission rate, low power consumption, low speed, large network capacity, small coverage, high reliability, flexible working frequency and flexible networking.

Cloud computing is an Internet based computing approach that provides services for businesses and individuals through the internet. Fundamentally speaking, cloud computing is based on the virtual technology as the core technology, the Internet as the carrier, the economies of scale driven, IT resource pool with a large amount of computing resources to provide virtualized IT service according to the demand of dynamic user.

The Development of Global Internet of Things. The use of the Internet of things is very extensive, involving the next generation of information networks and information resources control 
and utilization, and is expected to become one of the most important tools to manage the world. At present, all the countries are studying it deeply, and our government pays more attention to its construction. Since 2009 the United States put forward the concept of "the wisdom of the earth", a typical intelligent solution has been extended, the effect is to use the Internet of things change between the company and government and people communicate with people. All walks of life in the United States have attached importance to the research of the Internet of things, hoping to push forward the development of the Internet through innovation and technology improvement. In 1991, Japan and South Korea put forward the concept of "ubiquitous computing", and put forward the idea of building "ubiquitous network".

In 2009, when Prime Minister Jiabao Wen came to the Wuxi micro Nano sensor network engineering technology research center to inspect and make important speech, in August 24th of the same year, China Mobile President Jianzhou Wang went to Taiwan to make a public speech, puts forward the concept of "Internet of things". Through the various objects on the short distance transmission equipment, the realization of communication and dialogue between objects, all of these objects linked to the Internet of things".

\section{Internet of Things and Mobile Communication Network}

Internet of Things and Mobile Network Convergence Strategy. The deployment of regional networking is composed of a large number of micro sensor nodes, forming a network system with multiple self-organizations through wireless, wired network coverage, from collecting the necessary information of the object region, and communicated to the information collection. The history of the development of material things from the point of view, integration of wireless communication network and sensor network is an inevitable trend. The ubiquitous nature of the mobile network, the coverage of the wide area and the intelligent management of the terminal, will become the most important link in the development of the Internet of things.

At present, the world's largest mobile network operator in the networking service to a strategic height, the domestic operators of things have a relatively leading research and development and it has made remarkable achievements in its products, at the same time also accumulated some experience. Existing products, the most widely used is the ubiquitous card.

The development strategy of Internet of things and mobile network operators mainly includes three levels. There are a lot of wireless sensor technology standards in the sensing layer, and there are many research institutions and professional companies in the study. Mobile network and the Internet of things perception layer is not much, more attention is paid to how the sensor network and communication network. The network layer includes an access unit and an access network, and the access network is a communication network. The era of Internet of things will produce a lot of communication, but also different from the communication between people and the world, will bring greater pressure on the communication network. Internet of things is also a lot of problems and requirements for the communication network. Application layer includes middleware and application. Middleware level can be combined with the communication network to bring more applications.

Internet of Things Business Model Analysis. The data model of the Internet of things is a packet, high-frequency data services, similar to our daily chat. The Internet of things data service is also similar to an online service, because of its large number, and to keep online, so it will waste a lot of network resources, resulting in low utilization of the network. The arrival of the era of the Internet of things, the data packet is smaller; the frequency will be higher, resulting in higher data 
load. If the use of the original network optimization will make the problem more obvious, so it will become the bottleneck of the operation of the company through the wireless networking services.

\section{LTE Wireless Communication Systems and Internet of Things}

The Internet of things needs to control the sensor and the sensor through the self-organizing network and other local area network to overlay the sensor, using LTE wireless network communication as a gateway to access, When the data flows through the self-organizing network, it will be very large when the input back to the LTE network, and it will also produce more small packets and high frequency data, which will cause great pressure on the wireless communication network.

LTE technology uses orthogonal frequency division multiplexing technology, the channel is divided into several sub channel data through the channel can be high-speed data stream into parallel low-speed data streams, which can be modulated to each sub channel for transmission, at the same time through the two layer of wireless resource scheduler in dynamic scheduling. In summary, you can make use of LTE technology makes it unique to the Internet packet data transmission business possible. In addition, from the point of view of the core network, the core network can be notified through the NAS message and the corresponding QCI to carry out the transmission of unlimited, through the access network to establish the relevant wireless bearer.

The core network of LTE communication system does not have the mechanism of active release, so it will not be released because of the lack of data. Only access to the network news or UE through the NAS notification of the core network will really release. From the perspective of access network, wireless access network parameters according to the parameters of the QCI core network configuration, but also because the LTE user transmission by adapting the shared channel resources, so the configuration is more flexible, resource occupancy rate will be reduced to the minimum.

The sharing of resources using OFDM LTE, with two layer for dynamic scheduling of resource scheduler, the signaling resource release, and resources will not affect the air, so can keep online.

Through the analysis of the existing data form of the Internet of things, we must optimize the LTE system in accordance with a variety of Internet of things data model, in order to make a variety of things to carry out normal business and ensure network stability. At this stage, if fully in accordance with the agreement, LTE can not meet the requirements of the Internet of things. But through the 3GPP message in a custom domain, get a new QCI, according to the specific packet and the high frequency data model of LTE communication systems are optimized to produce small, high-frequency things infinite bearing, better network service for material. With the continuous development of science and technology, the Internet of things technology, cloud computing and other technologies will inevitably become the mainstream of public services, LTE can better combine with the Internet of things, to provide faster, more stable network load.

\section{Problems and Future Trends in the Development of Internet of Things Communication Technology}

The Development of Communication Technology of Internet of Things. With the rapid development of information and network technology, networking technology has been rapid development and has extensive application and promotion, but also put forward higher requirements on the mobile communication technology carrying Internet communication business. In the next development of the Internet of things, there are a series of problems need to be solved. First, the pursuit of ubiquitous Internet of things, but there are still many problems in communication 
technology, such as short communication distance, the impact of the surrounding environment. Although 3G, LTE technology can solve part of the problem, but because of the small amount of communication packets, high frequency characteristics, will take up a large number of its original communication resources. Second, there are some problems in the integration of various communication technologies. There are many kinds of communication technologies in the Internet of things, and there are many kinds of access. In the future, with the development of information technology, more and more new communication technologies will be developed. This is bound to facilitate the expansion of the Internet of things, but how to coordinate different objects, different technologies and the rational allocation of resources and to avoid conflicts, which we need to consider. Third is the problem of communication speed. Accesses to large scale of things, and in the continuous expansion of the communication channel speed, bandwidth requirements are very high. At present, the communication technology cannot meet the needs of the Internet of things. Fourth is the Internet of things communication technology security problems. The communication technology of the Internet of things is mainly composed of wireless communication, At present, many wireless communication technology is still developing, such as the security protocol is not complete, the security mode is simple and so on.

Development Trend of Internet of Things Communication Technology. Communication technology is the key to the Internet of things, although there are some problems in the Internet of things, but from the development of information and network technology, these problems can be solved. In 1991, the concept of ubiquitous network is composed of intelligent network, advanced computing technology and information infrastructure, the goal is to achieve interoperability with any object can be conceived. With the development of information technology, Internet access equipment, sensor network and communication network become more complicated, the formation of the coexistence of different network structure of the situation, this will seriously affect the Internet of things are interconnected. The needs of different wireless network integration and puts forward suitable protocol architecture constitute a wireless communication network, communication technology with LTE or higher level as the carrier, to provide high quality service for the user. The technology of the future will provide the support for the integration of heterogeneous communication of the Internet of things, and solve the problem of protocol conflict. The arrival of the era of big data in the future, will make the Internet of things each device generates a large amount of data, data storage processing has also brought great challenges. Cloud computing and other ways to deal with data and distributed computing to support large-scale data processing in the Internet of things. Therefore, the future direction of development must be the development of large-scale data processing.

\section{References}

[1] T.F. Li, Y.J. Huang and Z. Zhen: Communications Technology, Vol.46 (2013) No.4, p.121. (In Chinese)

[2] X. Liu and Y. Liu: Communications Technology of Internet of Things (Tsinghua University Press, China 2014). (In Chinese)

[3] L.Y. Liu and M.T. Gong: Communications Technology, Vol.46 (2013) No.3, p.95. (In Chinese)

[4] Chui M, Loffler M and Roberts R: McKinsey Quarterly, Vol.2 (2010) No.1, p.1.

[5] Mulligan G: IEEE Internet Computing, Vol. 14 (2010), No.1, p.35. 